\title{
Design of passive directional acoustic devices using Topology Optimization - from method to experimental validation
}

\section{Christiansen, Rasmus Ellebæk; Fernandez Grande, Efren}

Published in:

Journal of the Acoustical Society of America

Link to article, DOI:

$10.1121 / 1.4967370$

Publication date:

2016

Document Version

Publisher's PDF, also known as Version of record

Link back to DTU Orbit

Citation (APA):

Christiansen, R. E., \& Fernandez Grande, E. (2016). Design of passive directional acoustic devices using Topology Optimization - from method to experimental validation. Journal of the Acoustical Society of America, 140(5), 3862-3873. https://doi.org/10.1121/1.4967370

\section{General rights}

Copyright and moral rights for the publications made accessible in the public portal are retained by the authors and/or other copyright owners and it is a condition of accessing publications that users recognise and abide by the legal requirements associated with these rights.

- Users may download and print one copy of any publication from the public portal for the purpose of private study or research.

- You may not further distribute the material or use it for any profit-making activity or commercial gain

- You may freely distribute the URL identifying the publication in the public portal 


\title{
Design of passive directional acoustic devices using Topology Optimization - from method to experimental validation
}

\author{
Rasmus E. Christiansen ${ }^{a)}$ \\ Section for Solid Mechanics, Department of Mechanical Engineering, Technical University of Denmark, \\ Building 404, Nils Koppels Allé, DK-2800 Kongens Lyngby, Denmark \\ Efren Fernandez-Grande \\ Acoustic Technology, Department of Electrical Engineering, Technical University of Denmark, Building 352, \\ Oersteds Plads, DK-2800 Kongens Lyngby, Denmark
}

(Received 20 May 2016; revised 18 October 2016; accepted 27 October 2016; published online 21 November 2016)

The paper presents a topology optimization based method for designing acoustic focusing devices, capable of tailoring the sound emission pattern of one or several sources, across a chosen frequency band. The method is demonstrated numerically considering devices optimized for directional sound emission in two dimensions and is experimentally validated using three dimensional prints of the optimized designs. The emitted fields exhibit a level difference of at least $15 \mathrm{~dB}$ on axis relative to the off-axis directions, over frequency bands of approximately an octave. It is demonstrated to be possible to design focusing devices of dimensions comparable to the acoustic wavelength, a frequency range which is typically problematic, as well as devices operating at higher frequencies. The classical parabolic reflector is used as a benchmark. The devices designed using the proposed method are shown to outperform the latter in terms of directivity and maximum side-lobe level over nearly an octave band. A set of frequencies are considered simultaneously in the design formulation and performance robustness toward uniform spatial production errors in the designed devices is assured by including perturbations of the geometry in the design formulation. (C) 2016 Acoustical Society of America. [http://dx.doi.org/10.1121/1.4967370]

[MRB]

Pages: $3862-3873$

\section{INTRODUCTION}

This paper proposes a method for designing directional sound emission devices, operating under free field conditions, based on the gradient based optimization technique of topology optimization. ${ }^{1}$ Cases of devices optimized to function in different frequency regions are provided along with the experimental validation of several devices. The agreement between the predicted performance and the experimental measurements underline the effectiveness of the model and method.

In acoustics, it is often of interest to generate directionally focused sound fields, i.e., sound fields where the waves are emitted in a specific direction, and the acoustic energy is spatially confined. The problem is of fundamental interest for sound field reproduction purposes, ${ }^{2-4}$ acoustic measurements, ${ }^{5}$ long range emission, ${ }^{6,7}$ noise control, and others. ${ }^{8}$ However acoustic perturbations will in the general case not result in a directional and spatially confined sound field. The problem is thus of relevance both for sound emission problems, and the reciprocal problem of acoustic reception/sensing, ${ }^{9}$ mostly concerned with enhancing the directional sensitivity of a measurement system.

The most common and perhaps most intuitive focusing device is the parabolic reflector, ${ }^{7,10,11}$ which is based on basic geometrical considerations that are valid when

\footnotetext{
a)Electronic mail: raelch@mek.dtu.dk
}

assuming ray propagation. Nonetheless, these considerations do not contemplate the actual wave behavior of sound waves observed at low frequencies, and fail to account for fundamental phenomena such as scattering and diffraction. As a result, these devices are effective at high frequencies and perform quite well in a wide frequency sense, but their effective directionality is compromised, particularly at low frequencies. A fundamentally different alternative is the use of active systems, where the phase reproduction via an array of loudspeakers can be used to achieve the wanted directivity. This requires the introduction of additional sources, which can give rise to unwanted artifacts due to constructive interference between the sources. ${ }^{12}$

Extensive work, which served as inspiration for the present work, has treated the shape and/or topology optimization of an acoustic horn, for improved transmission among other goals. ${ }^{13,14}$ Other work, Düring et al. ${ }^{15}$ treating the application of topology optimization to the problem of local pressure reduction also served as a basis for this work. Other works of interest concerning the application of topology optimization to problems in acoustics, focused on limiting sound emission include, Du and Olhoff ${ }^{16}$ and Kook et al. ${ }^{17}$

The proposed method allows for designing focusing devices that use multiple sources, in order to maximize the total power output of the device, without relying on active cancellation of the emitted fields, but rather on the focusing of the total energy emitted. The devices are designed using density based topology optimization, assuming free-field propagation (in two-dimensions) governed by the Helmholtz 
equation with suitable boundary conditions. The design problem is formulated as a minimization problem. The objective function being the difference, in a suitable measure, between the pressure field emitted by on or more acoustic sources and a prescribed target pressure field. A continuous design field, used to control the material distribution, is introduced. The objective function is minimized over the design field for a set of frequencies in a prescribed frequency band simultaneously (contrary to the earlier studies for interior acoustic problems in Christiansen et al. ${ }^{18,19}$ where single frequency problems were considered). The design field is subject to a double filtering and projection strategy. ${ }^{18}$ This is done, in part to decrease the sensitivity of the device toward uniform geometric perturbations, which might be encountered in production, and in part as the method result in designs consisting nearly purely of solid and air as the direct output from the design process, hereby limiting the need for postprocessing. The use of the continuous design field allows for the application of the mathematical programming based optimization algorithm GCMMA, ${ }^{20}$ to solve the minimization problem.

The method works by modifying the material distribution constituting the device. The final material distribution acts as a number of rigid surfaces disturbing, guiding and reflecting the pressure field, giving rise to changes in the acoustic path lengths inside the device, shaping interference patterns, as well as controlling the direction of propagation of the pressure field. This ultimately results in the emission of an approximation of the desired field.

Although the present work only considers a twodimensional (2D) problem the proposed design formulation can be extended to cylindrically symmetric problems by considering the appropriate partial differential equation (PDE), ${ }^{21}$ as well as full 3D problems, however with a significant increase in the computational efforts in solving the PDE-problem.

\section{THE DESIGN PROCEDURE}

The goal of the design procedure is to create devices capable of shaping the pressure field emitted by a number of point-like sources inside the device to approximate a specified target pressure field. The cases studied in the paper address the design of devices emitting locally plane wavefronts in the near field, resulting in directional far field emission. However, the proposed method can be used to design devices which generate other specific pressure fields as well.

\section{A. The model problem}

An exterior acoustic model problem is used as the basis for the design problem. It is defined on the domain, $\Omega \subset \mathbb{R}^{2}$, sketched in Fig. $1 . \Omega$ is truncated by the curve $\delta \Omega$, along which a far field matching condition is imposed to approximate the Sommerfeld radiation condition. Two sub-domains are defined on $\Omega$. These are the design domain, $\Omega_{\mathrm{d}}$ : the subdomain containing the directional sound emission device under design and the target domain, $\Omega_{\mathrm{op}}$ : the sub-domain on which the objective function to be minimized is defined. Finally a collection of $i \in \mathbb{N}$ point-like sources, denoted $\delta P_{i}$, are distributed in $\Omega_{\mathrm{d}}$ and modeled using a Neumann

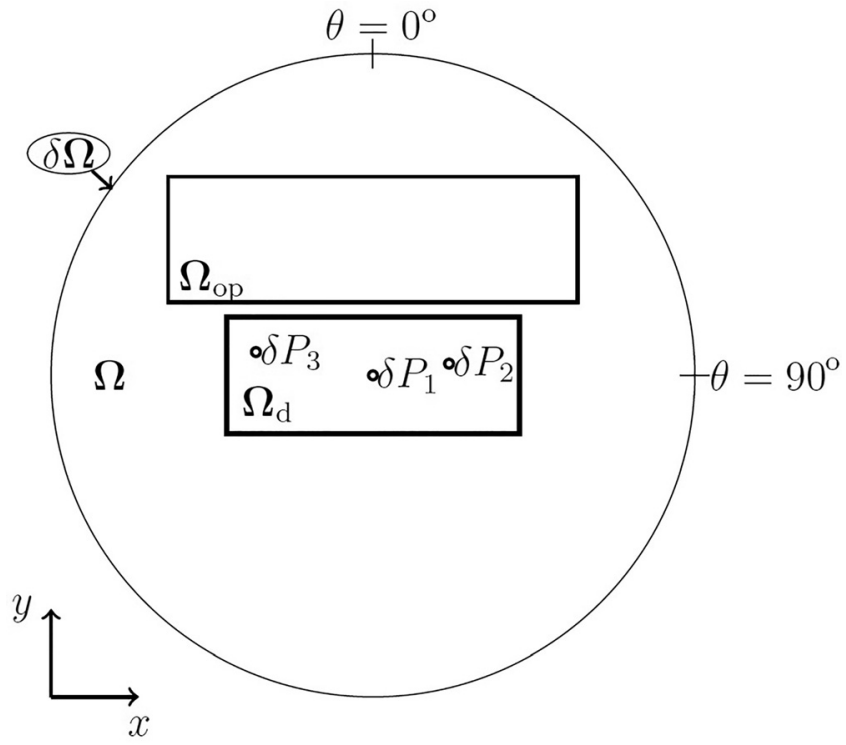

FIG. 1. Model problem sketch. $\Omega$ : Truncated Domain. $\delta \Omega$ : Truncation boundary. $\Omega_{\mathrm{op}}$ : Optimization domain. $\Omega_{\mathrm{d}}$ : Design domain. $\delta \mathbf{P}_{i}$ : Point-like sources. $\theta=0^{\circ}$ and $\theta=90^{\circ}$ : Denote the angular convention.

boundary condition allowing for an arbitrary shape and size of the sources if desired. The system is modeled using the Helmholtz equation and boundary conditions,

$$
\begin{aligned}
& \nabla \cdot\left(\rho(\mathbf{x})^{-1} \nabla p(\mathbf{x})\right)+\omega^{2} \kappa(\mathbf{x})^{-1} p(\mathbf{x})=0, \mathbf{x} \in \Omega, \\
& \lim _{|\mathbf{r}| \rightarrow \infty} \sqrt{|\mathbf{r}|} \cdot\left(\frac{\partial p(\mathbf{r})}{\partial|\mathbf{r}|}-\mathrm{i} \sqrt{\frac{\rho}{\kappa}} \omega p(\mathbf{r})\right)=0, \mathbf{x} \in \delta \Omega, \\
& \mathbf{n} \cdot \nabla p=-\mathrm{i} \rho \omega U, \mathbf{x} \in \delta P
\end{aligned}
$$

Here $\mathbf{x}$ and $\mathbf{r}$ denote the spatial dependence in Cartesian and Polar coordinates, respectively, and $\mathbf{n}$ denotes the normal vector; $\mathrm{i}$ is the imaginary unit, $p$ denotes the pressure field, $\omega=2 \pi f$ is the angular frequency and $f$ is the frequency. $U$ is the vibrational velocity of the source. $\rho(\mathbf{x})$ and $\kappa(\mathbf{x})$ are the density and bulk modulus, respectively, taking values of either solid material or fluid medium (air). The introduction of a solid material in $\Omega_{\mathrm{d}}$ would in the general case require a modification of the model problem to account for waves exited in the solid. However, in the present case, the choice of materials result in an impedance difference of more than three orders of magnitude between solid and air regions. Hence any waves excited in the solid will not perturb the pressure field in the air significantly and vise versa. This assertion has been validated using a full elasto-acoustic model in COMSOL Multiphysics $5.2 \mathrm{a}$ and through the experiments presented in the paper, (see also Christiansen et al. ${ }^{19}$ ).

\section{B. The design problem}

The design problem is formulated as a PDE-constrained optimization problem, for which the following objective function to be minimized is defined,

$$
\Phi=c_{s} \int\left(|p|^{2}-\left|p_{\text {target }}\right|^{2}\right)^{2} \mathrm{~d} \Omega_{\mathrm{op}} \geq 0 .
$$


Here $p_{\text {target }}$ describes the desired pattern of the sound pressure field emitted by the device and $c_{s}$ is a scaling parameter chosen to improve the convergence of the design process. $\Phi=0$ corresponds to a perfect match in pressure level between $p$ and $p_{\text {target }}$ everywhere in $\Omega_{\mathrm{op}}$.

For all cases treated in this paper the target field, $p_{\text {target }}$, is chosen to be a localized plane wave, $p_{\mathrm{EPW}}$, truncated by a Gaussian envelope, originating in and traveling away from $\Omega_{\mathrm{d}}$. For this choice of $p_{\text {target }}$, minimizing $\Phi$ in Eq. (4), may be thought of as maximizing the energy in the main lobe in front of the device while minimizing side lobe levels.

$$
\begin{aligned}
& p_{\text {EPW }}=A \mathrm{e}^{-\left(\left[\left(\mathbf{x}-\mathbf{x}_{w}\right) \cdot \hat{\mathbf{d}}\right] / \delta_{w}\right)^{2}} \mathrm{e}^{-\mathrm{i} \omega(\mathbf{x} \cdot \mathbf{d})} \mathrm{H}\left(\left(\mathbf{x}-\mathbf{x}_{w}\right) \cdot \mathbf{d}\right), \\
& \mathbf{d}=\left(\begin{array}{c}
\cos (\theta+\pi / 2) \\
-\sin (\theta+\pi / 2)
\end{array}\right), \quad \mathbf{x}_{w}=\left(\begin{array}{c}
x_{w} \\
y_{w}
\end{array}\right) .
\end{aligned}
$$

Here $A$ is the wave amplitude, $\mathbf{d}$ controls the propagation direction, $\hat{\mathbf{d}}$ denotes the transpose of $\mathbf{d}, \mathbf{x}_{w}$ is the position of the center of the wave, $\delta_{w}$ controls the width of the envelope and $\mathrm{H}(\cdot)$ denotes the Heaviside projection function. Note the untraditional angular convention illustrated in Fig. 1.

The amplitude of $p_{\text {EPW }}$ is selected by equating the power contained in $p_{\text {EPW }}$ to the power emitted from all sources placed in $\Omega_{\mathrm{d}}$, denoted $\mathrm{P}_{\mathrm{s}}$, and solving for $A$. The sound power emitted from a collection of time harmonic acoustic sources is calculated as, ${ }^{12}$

$$
\mathrm{P}_{\mathrm{S}}=\int_{S} \mathbf{n} \cdot \mathbf{I} \mathrm{d} \mathrm{S}=\int_{S} \mathbf{n} \cdot\left(\frac{1}{2} \Re\left(p \mathbf{u}^{*}\right)\right) \mathrm{dS},
$$

where $S$ denotes the surface, $\mathbf{n}$ is the surface normal, $\mathbf{I}$ is the time averaged sound intensity, $p$ is the pressure, $\mathbf{u}$ is the particle velocity, $\Re$ denote the real part and $(\cdot)^{*}$ denotes the complex conjugate.

The objective function, $\Phi$, is minimized by introducing an optimized design in $\Omega_{\mathrm{d}}$ consisting of a distribution of solid material placed in an air background. The distribution of the solid material is controlled by an auxiliary field $\xi(\mathbf{x})$ used to interpolate the material parameters, $\rho$ and $\kappa$ between solid and air as,

$$
\begin{aligned}
& \rho^{-1}=\rho_{\text {air }}^{-1}+\xi\left(\rho_{\text {solid }}^{-1}-\rho_{\text {air }}^{-1}\right), \\
& \kappa^{-1}=\kappa_{\text {air }}^{-1}+\xi\left(\kappa_{\text {solid }}^{-1}-\kappa_{\text {air }}^{-1}\right) .
\end{aligned}
$$

A detailed description of the optimization problem solved to minimize (4), the discretization of the design field, $\xi$, into a piecewise constant field of a finite number of design variables and the techniques used to regularize the design field, minimize the need for postprocessing and assure geometric robustness toward prescribed variations in the design is provided in Appendix A.

\section{The design domain}

The design domain $\Omega_{\mathrm{d}}$, is further divided into three subdomains, denoted; the designable $\Omega_{\mathrm{d}, \mathrm{d}}$, the empty $\Omega_{\mathrm{d}, \mathrm{e}}$, and the filled $\Omega_{\mathrm{d}, \mathrm{f}}$, sub-domain. $\Omega_{\mathrm{d}, \mathrm{d}}$ is the freely designable part of $\Omega_{\mathrm{d}}$, where material can be introduced and removed during the design procedure. $\Omega_{\mathrm{d}, \mathrm{e}}$ and $\Omega_{\mathrm{d}, \mathrm{f}}$ are fixed to be empty of and filled with solid material, respectively. In the numerical studies presented in the following, two configurations for $\Omega_{\mathrm{d}}$ are considered. These are illustrated in Fig. 2(a) and Fig. 2(b), respectively. Here, $\Omega_{\mathrm{d}, \mathrm{d}}$ is colored light gray while $\Omega_{\mathrm{d}, \mathrm{f}}$ is marked using dark gray and $\Omega_{\mathrm{d}, \mathrm{e}}$ is colored white. The first configuration seen in Fig. 2(a), henceforth denoted $\mathrm{C}_{1}$, consists of a fixed sub-domain with one edge shaped as a parabolic reflector with the acoustic source placed in its focal point and a fixed shielding in front of the point source. The boundary of the reflector is defined by $\mathrm{e}_{1}$ through $\mathrm{e}_{4}$ in (10),

$$
\begin{aligned}
& \mathrm{e}_{1}: y=\frac{0.8}{\mathrm{w}} x^{2}-0.25 \mathrm{w}, \mathrm{e}_{2}: y=-0.25 \mathrm{w}, \\
& \mathrm{e}_{3}: x=-0.5 \mathrm{w}, \mathrm{e}_{4}: x=0.5 \mathrm{w} .
\end{aligned}
$$

The second configuration seen in Fig. 2(b) and denoted $\mathrm{C}_{2}$, consists of the fixed sub-domain along three of the four edges of $\Omega_{\mathrm{d}}$ to help guide the sound and three point like

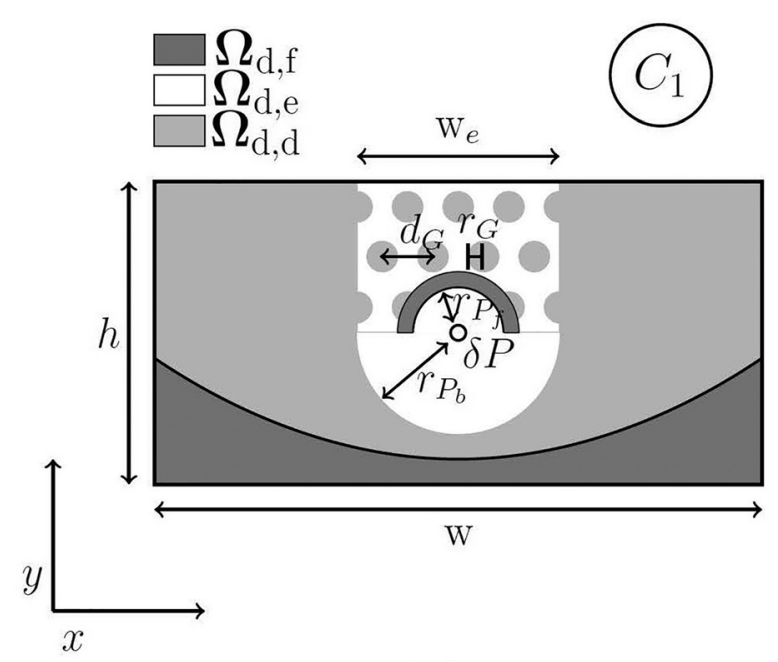

(a)

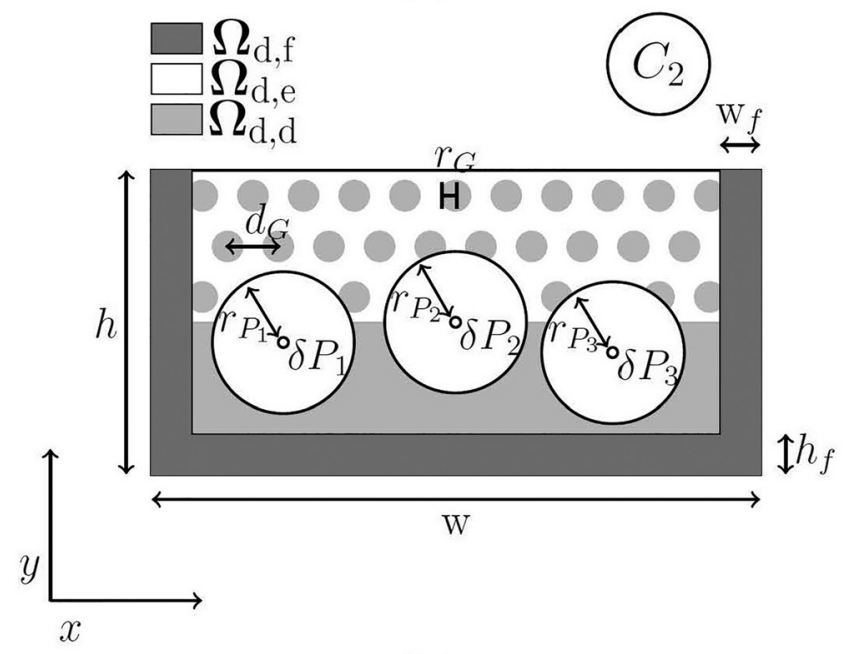

(b)

FIG. 2. Configurations of the design sub-domain, $\Omega_{\mathrm{d}}$. Filled sub-domain: $\Omega_{\mathrm{d}, \mathrm{f}}$. Empty sub-domain: $\Omega_{\mathrm{d}, \mathrm{e}}$. Designable sub-domain: $\Omega_{\mathrm{d}, \mathrm{d}}$. Domain width: w. Domain height: $h$. (a) Single source configuration, denoted $C_{1}$, with fixed parabolic reflector and shielding plate. (b) Three source configuration, denoted $C_{2}$, with fixed box along three of four edges. 
sources distributed inside $\Omega_{\mathrm{d}}$. For both configurations the origin is taken to be the center of $\Omega_{\mathrm{d}}$.

Through experimentation it was found that restricting $\Omega_{\mathrm{d}, \mathrm{d}}$ in front of the acoustic source(s) to an equiangular triangular grid, with grid size $d_{\mathrm{G}}$ and circular inclusions of radius $r_{\mathrm{G}}$, as illustrated by the light gray circles in Fig. 2, improved the reliability of the design procedure. The restriction makes it impossible for a large region of intermediate material/air to form in front of the source during the design process, which at the later stages of the design process risks turning into a block of solid material, as the projection strength in the continuation approach increases, effectively blocking the pressure field emitted from the device. (See Appendix A 2 regarding the continuation approach.) For both cases a circular region of radius $r_{P}$ around each acoustic source is fixed as air, i.e., belonging to $\Omega_{\mathrm{d}, \mathrm{e}}$. A number of additional parameters are used to define the different regions of $\Omega_{\mathrm{d}}$ as seen from Fig. 2 .

\section{IMPLEMENTATION AND PARAMETER CHOICES}

An implementation of the design procedure was written using MATLAB R2013a. A hybrid wave based and finite element method (hybrid WBM-FEM), ${ }^{22,23}$ was used to discretize the model equations. $\Omega_{\mathrm{d}}$ was discretized with the FEM using first order bi-linear finite elements. $\Omega \backslash \Omega_{\mathrm{d}}$ was discretized using the WBM. An impedance coupling was used to connect the FEM and WBM domains, described in chapter 7 of Pluymers. ${ }^{23}$ The Sommerfeld radiation condition was approximated using the proposal in chapter 6 of Pluymers. ${ }^{23}$ COMSOL Multiphysics $5.2 \mathrm{a}$ was used to validate the hybrid WBM-FEM solver. All numerical performance results presented in the following, was obtained using an acoustic model in COMSOL Multiphysics $5.2 \mathrm{a}$ on thresholded versions of the optimized devices.

The devices considered in the following have been designed using the parameter values provided in Table I. A total of $300 \times 150=45000$ quadrilateral first order finite elements are used to discretize $\Omega_{\mathrm{d}}$, matching the resolution of the $3 \mathrm{D}$-printer used to produce the devices used in the experimental validation.

\section{EXPERIMENTAL SETUP}

No design or design methodology can be fully trusted before it has been experimentally validated. With this in mind a two-dimensional anechoic chamber is built in order to provide a two-dimensional free-field space in which to test the designs. A schematic of the experimental setup is provided in Fig. 3(a) and a picture of the setup in Fig. 3(b) including a test specimen and the flush mounted microphone. The dimensions of the chamber and specifications regarding the components as well as relevant material parameters may be found in Table II in Appendix B. The chamber is constructed using a PVC plate as a bottom-plate, with wooden strips at its edges, and an acrylic plate as a top-plate, resting on the wooden strips. Open cell absorbing foam strips of $0.13 \mathrm{~m}$ width, made from melamine resin [Basotec (BASF, Germany)], with $6.5 \mathrm{~cm}$ deep wedges cut into them, are placed along the wooden strips. The limited height of the chamber ensures that only a two dimensional sound field can propagate in the chamber below the cutoff frequency, $f_{\text {cutoff }} \approx 28.5 \mathrm{kHz}$ (assuming a speed of sound of
TABLE I. Parameters used in the design problems.

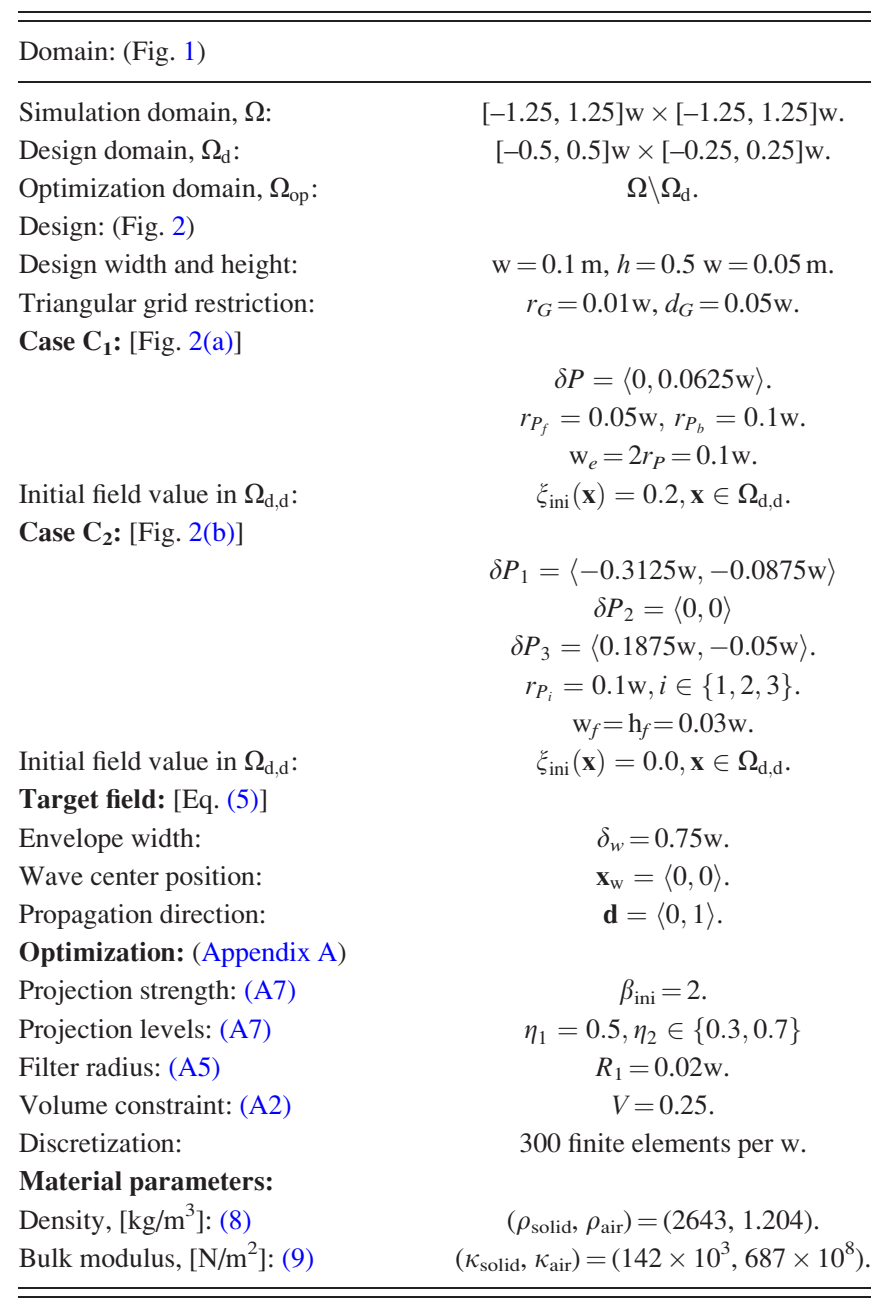

$c_{\text {sound }}=343 \mathrm{~m} / \mathrm{s}$ ). The source used is a 6 in. loudspeaker connected to a funnel and a wave guide which is connected to a hole in the specimen under test, effectively acting as an approximate point source. A circular hole is drilled in the center of the top-plate allowing for the flush mounting of an $\frac{1}{8}$ in. microphone [Brüel and Kjær (Naerum, Denmark)]. The microphone is connected to the data acquisition system, a NEXUS pre-amplifier and a PULSE analyzer manufactured by Brüel and Kjær (Naerum, Denmark), and a computer for data acquisition. The measurements are performed as follows. A test specimen is placed in the chamber and the wave guide attached appropriately. The source is driven with pseudorandom noise bandlimited to $6.4 \mathrm{kHz}$. The flush mounted microphone is scanned manually along the measurement points: a half circle of radius $r_{c}=0.22 \mathrm{~m} \pm 10^{-3} \mathrm{~m}$, centered at the hole in the test specimen. The pressure field is recorded using the microphone with $1 \mathrm{~Hz}$ spectral resolution and 20 averages. The angular resolution of the scanning is $2.5^{\circ}$ from $\theta=0^{\circ}$ to $\theta=180^{\circ}$. The uncertainty in the microphone placement is estimated to $\approx 1 \mathrm{~mm}$ in both spatial directions.

A reference measurement on a test specimen without a design (i.e., a point source in the empty chamber) is performed for calibration purposes and to investigate the field in the cavity. For frequencies, $f>4.8 \mathrm{kHz}(\mathrm{w} / \lambda \gtrsim 1.4)$, a maximal variation in the pressure of $2 \mathrm{~dB}$ sound pressure level 


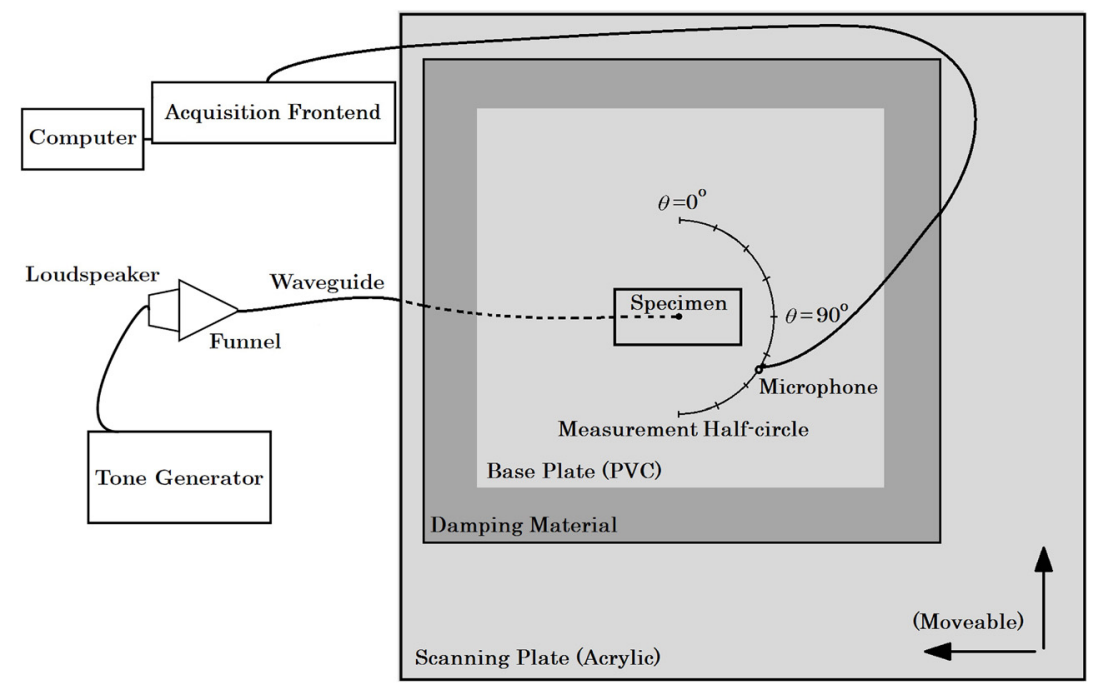

(a)

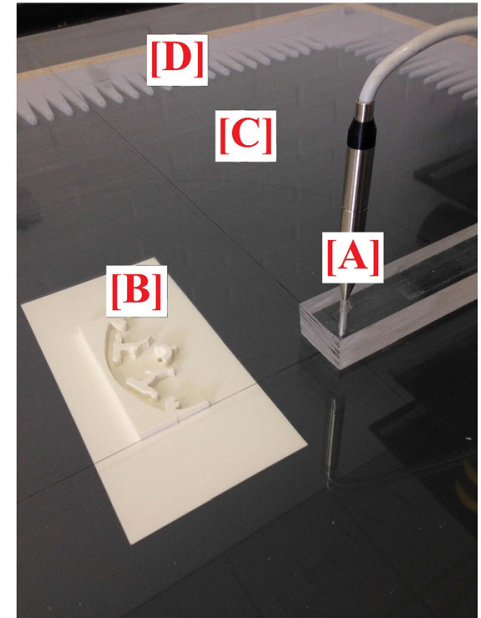

(b)

FIG. 3. (Color online) (a) Schematic of the experimental setup including the chosen angular convention $\left(\theta=0^{\circ}\right.$ in front of the test specimen). (b) Picture of the anechoic chamber, $[\mathrm{A}]$ the microphone, $[\mathrm{B}]$ a test specimen, $[\mathrm{C}]$ the PVC base plate under the acrylic scanning plate and [D] the absorbing foam and wooden strips.

(SPL) as a function of angle $\left(\theta \in\left[0^{\circ}, 180^{\circ}\right]\right)$ is observed. For $3.4 \mathrm{kHz}<f<4.8 \mathrm{kHz}$ a maximal variation in pressure of $3 \mathrm{~dB}$ SPL is observed and for $f<3.4 \mathrm{kHz}$ a maximal variation of $6 \mathrm{~dB}$ SPL is observed. The background noise in the chamber is measured at $\theta=0^{\circ}$ across the full frequency spectrum considered. On the basis of the measurement a lower limit on all subsequent measurements is defined to be $3 \mathrm{~dB}$ SPL above the background. All measured data points below this limit are removed, and for the visualization of the results replaced with dark gray [e.g., see Fig. 8(k)].

\section{RESULTS}

Two measures are used to evaluate the devices. The first is the far field sound pressure as a function of angle, $\theta$, relative to the far field sound pressure at $\theta=0^{\circ}$,

$$
P_{m}(\theta, f)=20 \log _{10}\left(\frac{\left|p_{\mathrm{far}}(\theta, f)\right|}{\left|p_{\mathrm{far}}\left(\theta=0^{\circ}, f\right)\right|}\right) .
$$

The far field pressure, $p_{\mathrm{far}}(\theta, f)$, is approximated using the Kirchoff-Helmholtz integral equation, e.g., see Appendix A. 2 of Bai et al. ${ }^{9}$ The second measure is the far field sound pressure on axis $\left(\theta=0^{\circ}\right)$ relative to the far field of a monopole radiating identical power into free space,

$$
\Delta L_{\mathrm{dB}}(f)=20 \log _{10}\left(\frac{\left|p_{\text {far }}\left(\theta=0^{\mathrm{o}}, f\right)\right|}{\left|p_{\text {monopole, far }}(f)\right|}\right) .
$$

\section{A. Reference: The parabolic reflector}

The parabolic reflector is a well known and frequently used design for directional emission/reception in both acoustics and electromagnetics. Assuming ray based propagation for the sound field and using geometric arguments it is easy to show that the parabola is an optimal solution for converting the field impinging on it from a point source placed at its focal point to a localized near-plane wave, as illustrated in Fig. 4(a). However, the fact that the ray-based model is only strictly valid for $f \rightarrow \infty$ and disregard fundamental wave propagation phenomena, opens a potential for design improvement. Another obvious limitation of the reflector is that it is ill-suited if several sources are considered.

A parabolic reflector of width $w$, described by the equations in (10), with a shielding in front of the source identical to the one for $C_{1}$ in Sec. IIC, is considered as a reference, $R_{p}$. Figure $4(\mathrm{~b})$ shows the design field for $R_{p}$ with a source at its focal point $(\delta P=\langle 0,0.0625 \mathrm{w}\rangle)$, illustrated by a red circle. Figure 4(c) shows the calculation of $P_{m}(\theta, f)$ for the parabolic reflector, $R_{p}$. It is observed that for $\mathrm{w} / \lambda<1.5$ the directivity of $R_{p}$ is poor and that for $1.1<\mathrm{w} / \lambda<1.5$ the side lobes and main lobe are seen to have equal value of $P_{m}$.

\section{B. A low frequency device}

As a first example of applying the proposed method, consider the problem of designing a device, with a width approximately equal to the wavelength in the frequency range where the device is designed to operate. This design problem is denoted $D_{1}$. The central frequency of operation, $f_{c, D_{1}}$, is chosen to correspond to the wavelength $\lambda_{1} \approx 0.89 \mathrm{w}$, and a $\Delta f_{D_{1}}=50 \%$ frequency band centered at $f_{c, D_{1}}$ is considered. The design process is executed using $i=9$ equidistant frequency values in $f \in\left[f_{c, D_{1}}-0.5 \Delta f_{D_{1}}, f_{c, D_{1}}+0.5 \Delta f_{D_{1}}\right]$ and $j=2$ projection levels, $\eta_{2} \in\{0.3,0.7\}$.

Figure 5 shows a convergence plot for the design process for $D_{1}$, using a total of 333 design iterations. It presents $\Phi_{i, j}$ as a function of design iteration number for all, $i \cdot j=18$ realizations. The maximum value of $\Phi$ across the 18 realizations for each design iteration is marked with 


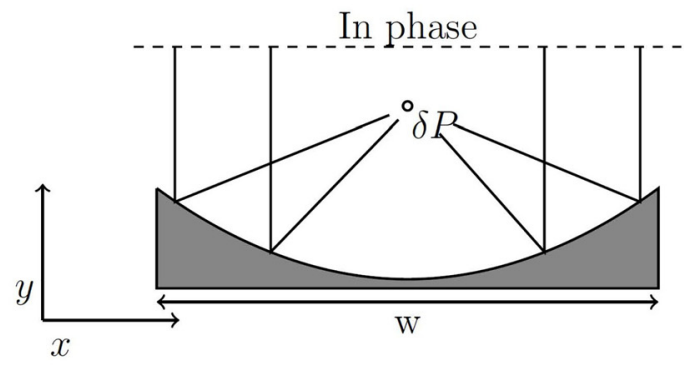

(a)

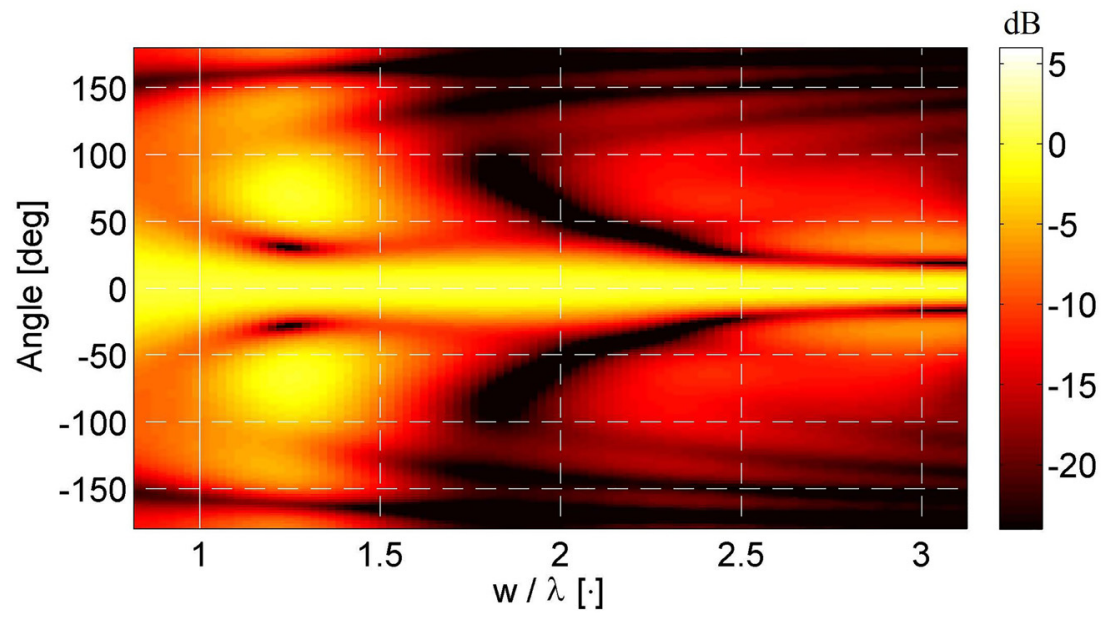

FIG. 4. (Color online) (a) Ray-based model for pressure field emitted by point source placed in front of a parabolic reflector. (b) Design field for reference parabolic reflector $R_{P}$. (c) Performance measure $P_{m}(\theta, f)$ for $R_{p}$ measured in $\mathrm{dB}$. The first axis indicate the parabola width $w$, relative to the wavelength in air, $\lambda$.

\section{(c)}

a black square. It is observed that the active objective function value, $\max _{i, j}(\Phi)$, changes several times during the optimization. The iteration numbers at which the $\beta$-value for the projection scheme increased are indicated in the figure using gray vertical lines. Jumps in the objective value are observed at semi-regular intervals. The jumps coincide with increases in the $\beta$-value for the projection operation in all but one case. These jumps are to be expected since

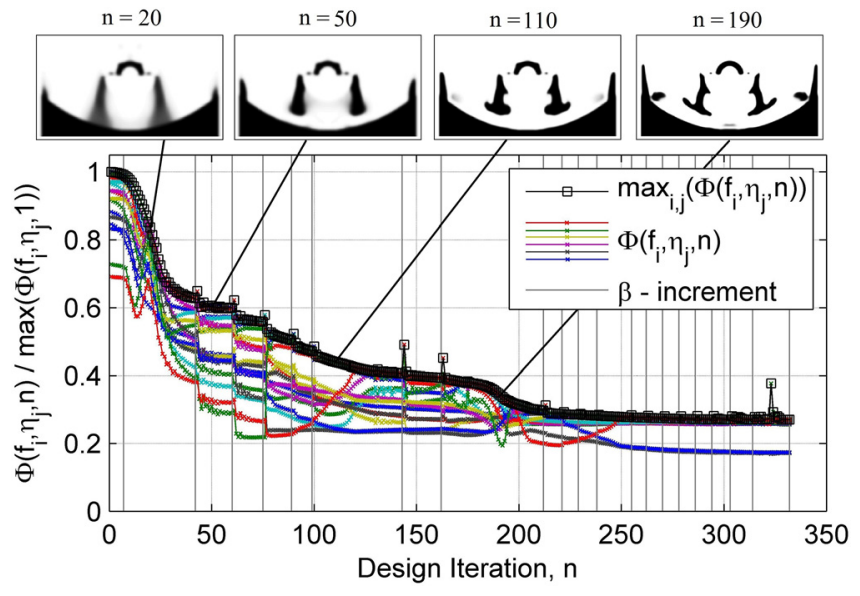

FIG. 5. (Color online) (Crosses) Normalized objection function value as a function of design iteration, $n$, for the $i \cdot j=18$ realizations. (Black squares) $\max _{i, j}(\Phi)$ at each iteration. (Gray vertical lines) $\beta$-increments. Snapshots of the filtered design field at the iterations $n \in\{20,50,110,190\}$ are included to illustrate the design evolution. increasing the $\beta$-value, i.e., steeper projection, causes an abrupt change in the design which in turn affects the objective value.

\section{Design}

The final design field, $\hat{\tilde{\tilde{\xi}}}\left(\eta_{j}\right), j \in\{1,2\}$, for the two projection levels used in the optimization, are presented in gray scale in Figs. 6(a) and 6(b), respectively. Black indicates solid material and white indicates air and a red circle is included to illustrate the position of the point-like source. As is the point of applying the robust approach small variations are observed around each design feature for the designs in Figs. 6(a) and 6(b), showing that the design has indeed been optimized to function under a small uniform geometric variation. The output of the design procedure is seen to consist almost exclusively of solid and air regions. A postprocessing step is performed on the design in Fig. 6(b), where the smallest features removed as seen in Fig. 6(c). The effect of the feature removal on the sound field was found to have negligible impact on the designs performance, while in turn improving producibility.

\section{Numerical performance}

Figures 7(a) and 7(b) show the performance measure $P_{m}(\theta, f \sim \mathrm{w} / \lambda)$ Eq. (11), for the postprocessed design in Fig. 6(c) for the design problem, $D_{1}$, and for the reference parabolic reflector, $R_{p}$, respectively. The frequency interval 


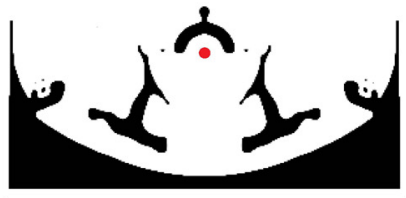

(a)

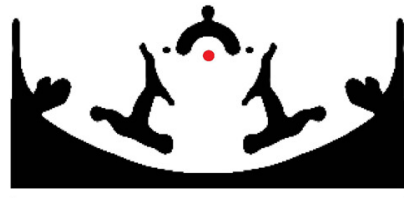

(b)

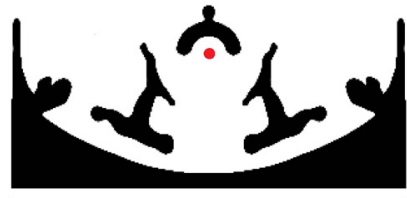

(c)
FIG. 6. (Color online) Design field, $\hat{\xi}$. (a) Design field projected at $\eta_{2}=0.7$. (b) Design field projected at $\eta_{2}=0.3$. (c) Postprocessed design field projected at $\eta_{2}=0.3$ using Heaviside projection. Black corresponds to solid material and white corresponds to air. for which $D_{1}$ was optimized is marked by white vertical lines and a green bar in both figures. From Fig. 7(a) it is seen that in the frequency interval for which $D_{1}$ was optimized, the main lobe (centered at $\theta=0^{\circ}$ ) contains most of the emitted

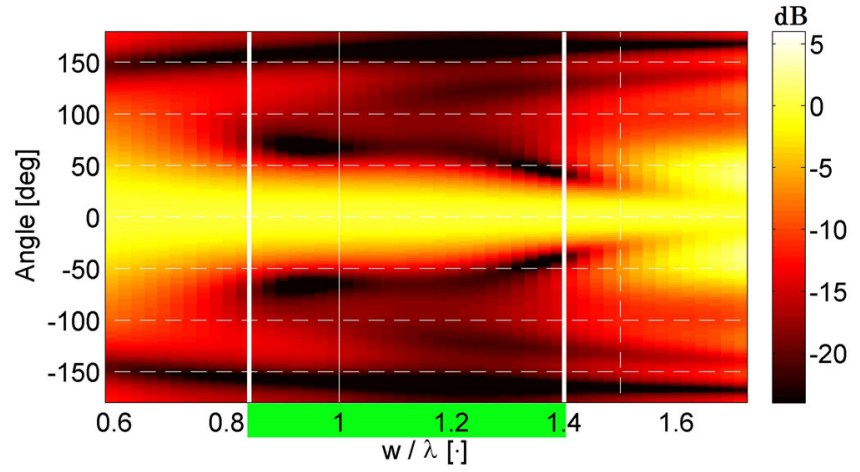

(a)

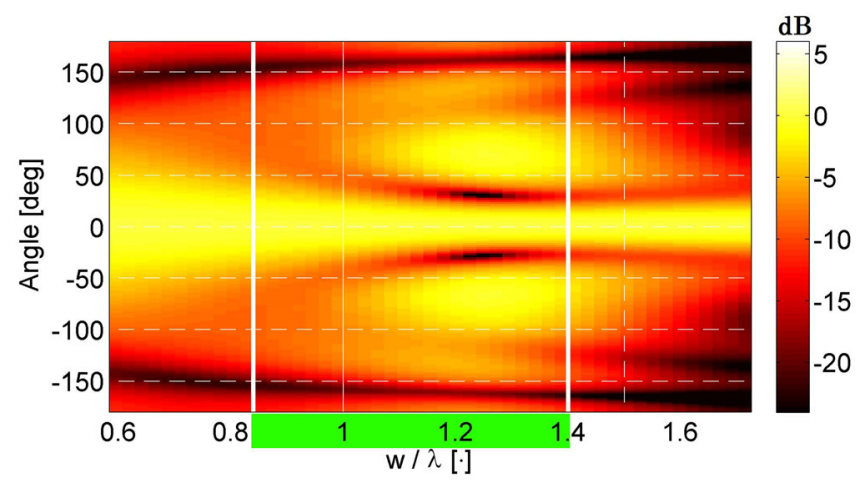

(b)

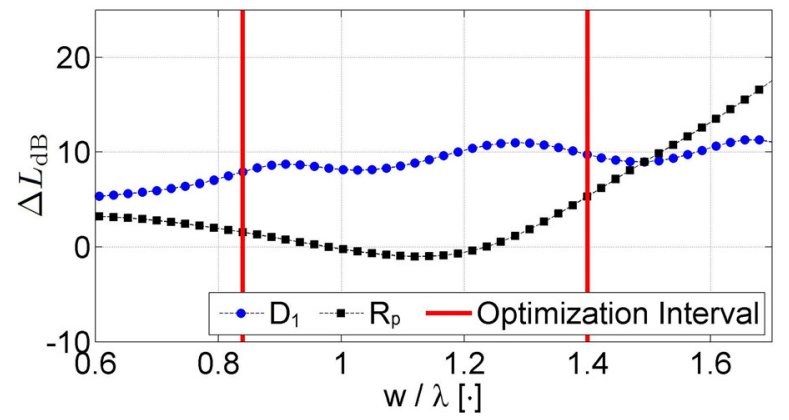

(c)

FIG. 7. (Color online) (a)-(b) Performance measure, $P_{m}(\theta, f)$, for (a) the optimized design, $D_{1}$, (b) the reference reflector, $R_{p}$. The vertical white lines and green bars denotes the boundaries of the frequency interval considered in the design problem $D_{1}$. (c) $\Delta L_{\mathrm{dB}}$ for (blue circles) $D_{1}$ and (black squares) $R_{p}$. Vertical red lines denotes the boundaries of the frequency interval considered in the design problem $D_{1}$. sound. In fact the pressure in the main lobe is more than $15 \mathrm{~dB}$ higher than in the rest of the angular interval. In contrast when considering the result for the reference reflector in Fig. 7(b), it is seen that the pressure in the main lobe is similar to the pressure in the side lobe (centered at $\theta \approx 75^{\circ}$ ), and that the reflector generally emits a higher sound pressure in all directions outside of the main lobe $\left(|\theta|>35^{\circ}\right)$. Hence across the interval of optimization the optimized design clearly outperforms the reflector in terms of directivity.

The increase in $\Delta L_{\mathrm{dB}}$, Eq. (12), is shown in Fig. 7(c) for $D_{1}$ (blue circles) and $R_{p}$ (black squares). The optimization interval is highlighted using vertical lines. It is seen that the optimized design, $D_{1}$ emits a higher sound pressure than the reference, $R_{p}$, across the full optimization interval. The increase in sound pressure ranges from 5 to $10 \mathrm{~dB}$. In addition the variations in the pressure level are smaller. Variations of $\approx 3 \mathrm{~dB}$, are observed for $D_{1}$ compared to $\approx 6 \mathrm{~dB}$ for $R_{p}$.

\section{Considering higher frequencies}

Two additional design problems, denoted $D_{2}$ and $D_{3}$, are considered to demonstrate the effectiveness of the proposed method over a wider frequency range. The central frequencies for $D_{2}$ and $D_{3}$ are denoted $f_{c, D_{2}}$ and $f_{c, D_{3}}$, and correspond to the wavelengths, $\lambda_{2} \approx 0.54 \mathrm{w}$ and $\lambda_{3} \approx 0.38$ $\mathrm{w}$, respectively. Frequency bands of $\Delta f_{D_{2}}=50 \%$ and $\Delta f_{D_{3}}$ $=30 \%$ centered at $f_{c, D_{2}}$ and $f_{c, D_{3}}$ are considered. Like for $D_{1}$, the design processes are executed using $i=9$ equidistant frequency values and $j=2$ projection levels.

\section{Designs}

Postprocessed versions of the design fields for the devices obtained for the design problems $D_{1}, D_{2}$, and $D_{3}$ are presented in Figs. 8(a)-8(c). Test specimens used in the experimental investigation of the devices, were manufactured in ABS plastic using 3D-printing, based on extruded versions of the postprocessed design fields. Images of the 3D-printed specimens are provided in Figs. 8(d)-8(f). The specimens consist of a base plate with the extruded device on top. The base plate has the dimensions of the hole in the PVC base plate in the experimental chamber, see Fig. 3(b), while the extruded device is printed with an precision of $0.2 \mathrm{~mm}$ (the resolution of the 3D-printer).

\section{Numerical performance}

The directivity measure, $P_{m}$, for the three devices obtained for the design problems $D_{1}, D_{2}$, and $D_{3}$ is presented in Figs. 8(g)-8(i). Vertical white lines and green 


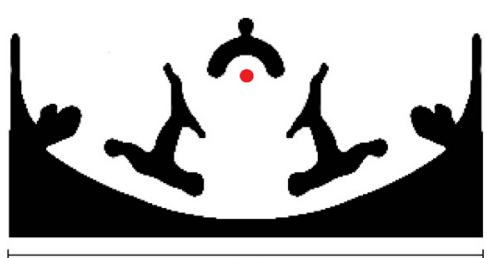

(a)

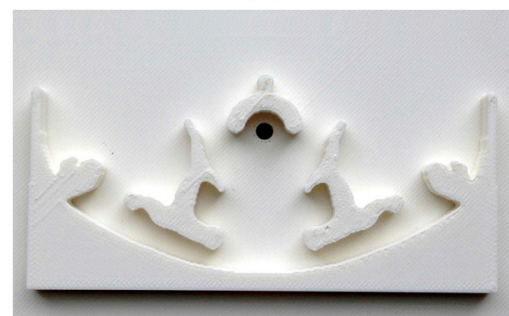

(d)

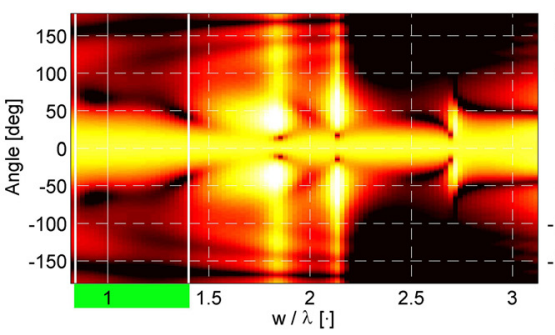

(g)

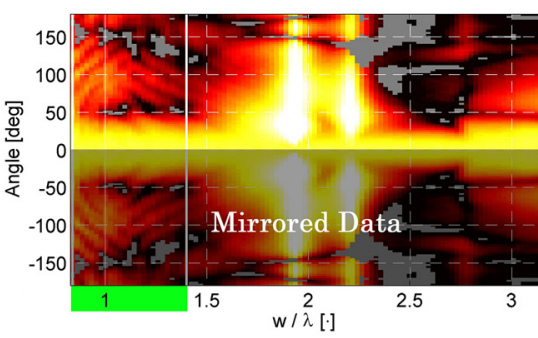

(j)

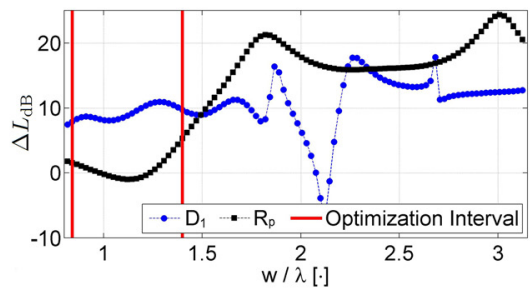

(m)

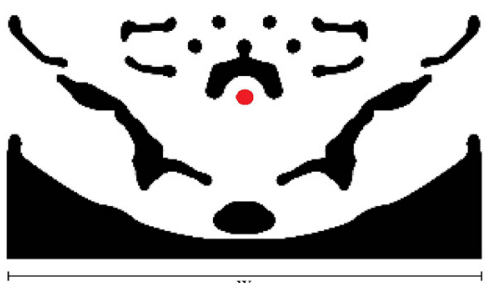

(b)

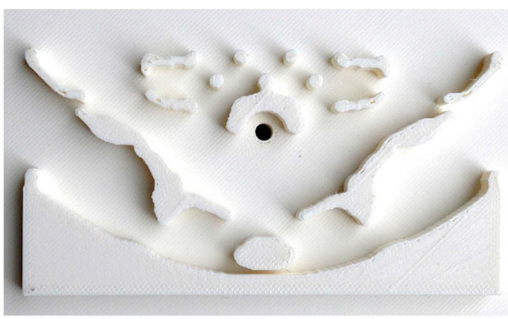

(e)

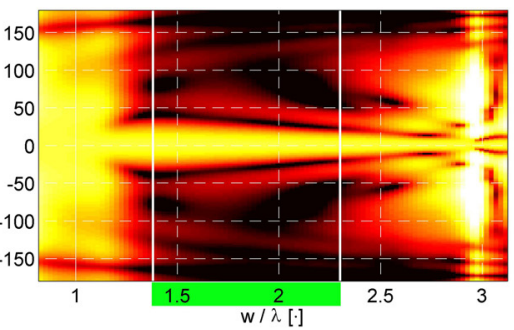

(h)

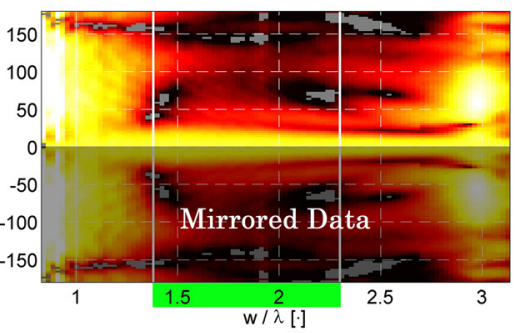

(k)

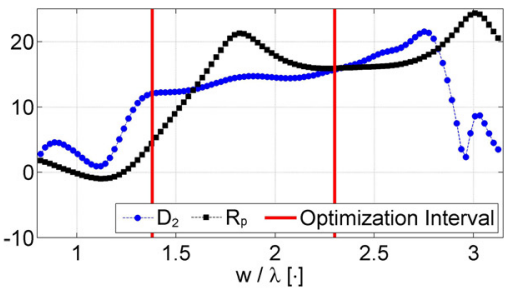

(n)

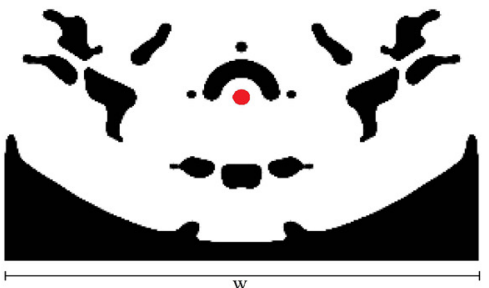

(c)

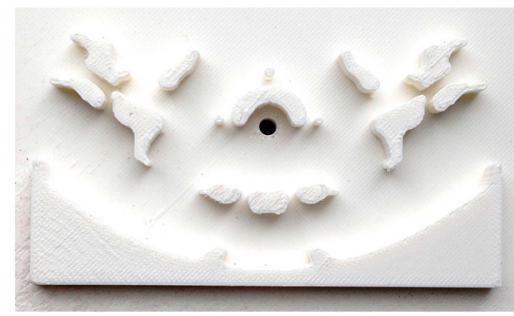

(f)

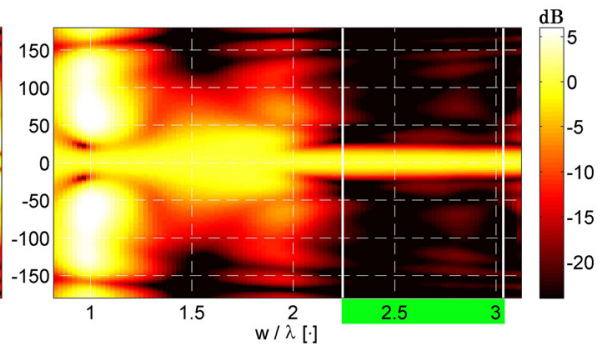

(i)

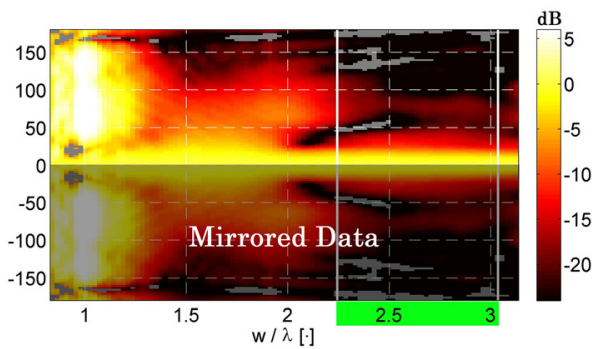

(l)

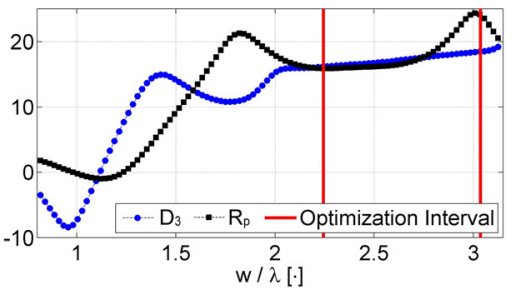

(o)

FIG. 8. (Color online) (a)-(c) Post processed design field for $D_{i}$, (a) $i=1$, (b) $i=2$, (c) $i=3$. (d)-(f) 3D-printed test specimen for $D_{i}$, (d) $i=1$, (e) $i=2$, (f) $i=3$. (g)-(i) $P_{m}(\theta, f)$ calculated for the postprocessed design field for $D_{i},(\mathrm{~g}) i=1$, (h) $i=2$, (i) $i=3$. (j)-(l) Experimentally measured pressure at $r \approx$ $0.22 \mathrm{~m}$, scaled by the on axis measured pressure $(\theta=0)$ in $\mathrm{dB}$, for the test specimens for $D_{i}$, (j) $i=1,(\mathrm{k}) i=2$, (1) $i=3$. For (g) $-(\mathrm{l})$ white lines and green bars illustrate the boundaries of the frequency bands considered in the design problems. (m)-(o) $\Delta L_{\mathrm{dB}}$ calculated for $R_{p}$ (black squares) and the postprocessed design field for $D_{i},(\mathrm{~m}) i=1$, (n) $i=2$, (o) $i=3$ (blue circles). Vertical red lines illustrate the boundaries of the frequency bands considered in the design problems.

bars are used to indicate the frequency interval across which the devices are designed to operate. For all three cases it is seen that, in these frequency intervals, the main lobe contains most of the emitted energy. Comparing across the three cases it is seen that both the fraction of the energy in the main lobe and the directivity increase from design $D_{1}$ to design $D_{2}$ and again from design $D_{2}$ to design $D_{3}$. All three devices outperform the reference parabolic reflector 
with respect to directivity as seen by comparing with Fig. 4(c). The performance increase is most evident for design $D_{1}$ and design $D_{3}$ since the reflector already performs well in the frequency interval where design $D_{2}$ is optimized to operate. An important and obvious observation is that the directivity for all three devices deteriorate significantly outside the frequency range where they are optimized to operate, limiting the devices to be used inside the considered frequency ranges.

Figures $8(\mathrm{~m})-8(\mathrm{o})$ present $\Delta L_{\mathrm{dB}}$ for the designs $D_{1}-D_{3}$. The frequency intervals for which the devices are designed to operate are highlighted using vertical lines. Considering these intervals, it is seen that $\Delta L_{\mathrm{dB}}$ varies less for the three designs than it does for the reference, $R_{p}$. Furthermore it is seen that $\Delta L_{\mathrm{dB}}$ increases with frequency, in agreement with the increasing directivity observed in Figs. 8(g)-8(i). Outside the frequency intervals, where the devices were designed to operate, $\Delta L_{\mathrm{dB}}$ is seen to decrease and/or vary rapidly for all three designs.

\section{EXPERIMENTAL VALIDATION}

The magnitude of the pressure field was measured experimentally, across $f \sim \mathrm{w} / \lambda \in[0.8,3.15]$ and $\theta \in\left[0^{\circ}\right.$, $180^{\circ}$ ], for each of the three test specimens in Figs. 8(d)-8(f), using the procedure described in Sec. IV. The results of the measurements are reported in Figs. 8(j)-8(1) using the measure $P_{m}$ from (11), with the far field pressure, $\left|p_{\text {far }}\right|$, replaced by the measured pressure, $\left|p_{\text {meas }}\right|$.

Consider first the experimental results on their own. Oscillations in the measured pressure, as a function of both $\theta$ and $\mathrm{w} / \lambda$, are clearly observed for $\mathrm{w} / \lambda<1.4$ and are observed to decrease with increasing frequency. This observation is in agreement with the findings from the reference measurement discussed in Sec. IV. That is, the damping material along the edges of the chamber is observed to have poorer absorption characteristics in the lower frequency range. It is further observed that in regions with low values of $P_{m}$, the limit of $3 \mathrm{~dB}$ above background noise is reached for all three designs.

A remarkable agreement is found when comparing simulations and experiments. Consider here the measured data in Figs. 8(j)-8(1) and the numerical data in Figs. 8(g)-8(i). Note that the range of the data is identical, i.e., going from $-24 \mathrm{~dB}$ to $6 \mathrm{~dB}$. The major features and variations observed in the numerical data are also found in the experimental data. That is, agreement is found in the magnitude and width of the main lobe, as well as in the distribution of low and high values of $P_{m}$ in the parameter domain, for all three designs. The largest deviations are found for $\mathrm{w} / \lambda<1.4$ and are, as mentioned in the previous paragraph, believed to stem from the poor absorption characteristics of the damping material in the lower frequency range. That is, the disagreement stems from experimental errors due to reflections from the boundaries.

\section{A. Multiple source device}

As a final demonstration of the generality of the proposed method, consider the problem of designing a device

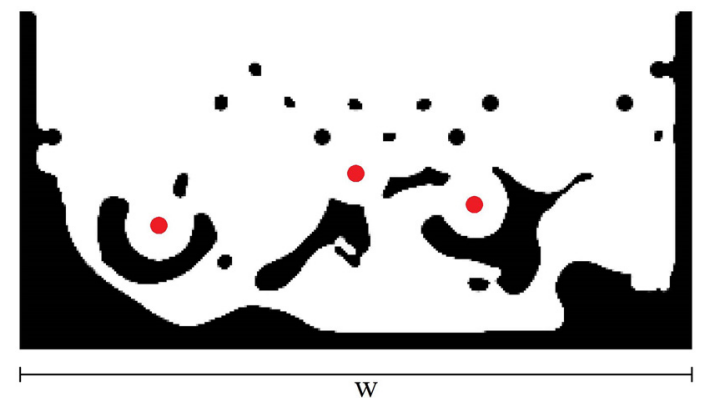

(a)

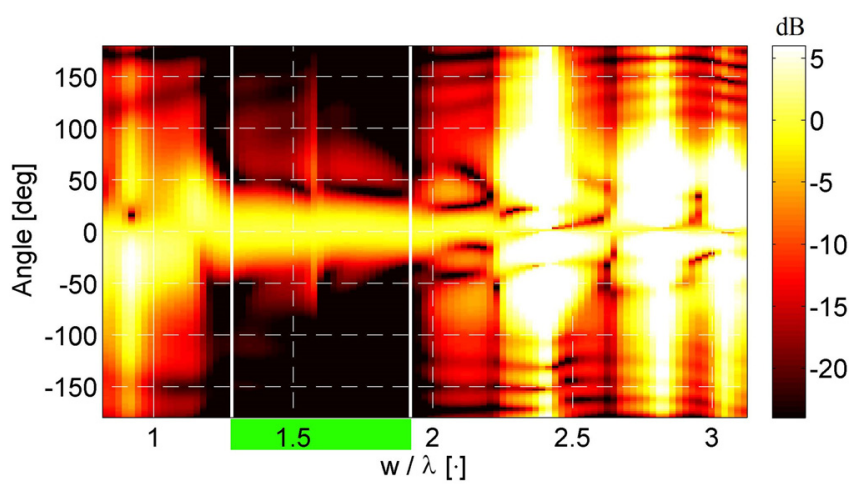

(b)

FIG. 9. (Color online) (a) Post processed design field for the device obtained in the design problem $D_{4}$ including red circles denoting the position of the point like sources. (b) $P_{m}(\theta, f)$ for the device obtained in the design problem $D_{4}$, including vertical white lines and a green ar to illustrate the boundaries of the frequency interval considered in the design problem.

for directional sound emission which contain three point-like sources placed asymmetrically inside $\Omega_{\mathrm{d}}$ as sketched in Fig. 2(b). This design problem is denoted $D_{4}$. The central frequency for $D_{4}$, denoted $f_{c, D_{4}}$, corresponds to the wavelength $\lambda_{4} \approx 0.625 \mathrm{w}$. A frequency band $\Delta f_{D_{4}}=40 \%$ centered around $f_{c, D_{4}}$ is considered. The design process is executed using $i=9$ equidistant frequency values and $j=2$ projection levels.

The postprocessed design for $D_{4}$ is presented in Fig. 9(a) with black being solid material and white being air. As for the illustrations of the design fields for the devices $D_{1}-D_{3}$ red circles are used to denote the position of the sources.

Figure 9(b) shows $P_{m}$, obtained numerically for $D_{4}$ in the intervals $\theta \in\left[-180^{\circ}, 180^{\circ}\right]$ and $\mathrm{w} / \lambda \in[0.8,3.15]$. The asymmetry of the problem is clearly observed in the figure. The interval for which $D_{4}$ is optimized is marked by white vertical lines and a green bar. In this frequency interval, the main lobe is seen to contain most of the emitted energy. A sudden increase in $P_{m}$ is observed at $\mathrm{w} / \lambda \approx 1.6$. This value corresponds to a frequency not considered in the optimization, suggesting that including more frequency values in the optimization would remove the jump. Just as for the designs obtained for $D_{1}-D_{3}$ it is observed that in several regions outside the interval for which $D_{4}$ was optimized to function its performance drops below that of the reference reflector, $R_{p}$. 


\section{CONCLUSIONS}

In summary, the paper has presented a topology optimization based design method for creating acoustic focusing devices exhibiting high directivity and emission efficiency in unbounded domains, considering a set of frequencies in the design process. The method is demonstrated in $2 \mathrm{D}$ using the parabolic reflector design as a reference. It is shown that the method is capable of designing devices functioning across broad frequency bands, at wavelengths comparable to the device dimensions, a frequency range which is typically problematic. An experimental approach and setup for validating the optimized devices is presented. Three designed devices were 3D-printed and their performance investigated using the experimental setup. The measured and numerical data show remarkable agreement, supporting the "real world" applicability of the method (barring implementing a 2D circular symmetrical or 3D version of the code). While the majority of the considered cases focused on the parabolic like reflector, the method is not restricted to this case. This was demonstrated by considering an alternate configuration with three point sources scattered asymmetrically inside the design domain. Other configurations and more complex near fields may be considered with the method by simple modifications of the target field and/or design domain.

As a final remark it is noted that relatively small changes in the choice of model parameters have been found to result in large changes in the geometry of devices obtained using the proposed method. In all cases the changes in device geometry have been found to have limited effects on the performance of the device however and are thus not deemed problematic. The reason behind the observed sensitivity toward parameter choices is believed to be the nature of the design problem, having a vast design space, coupled with the nature of the acoustic problem. The finding is in agreement with the conclusion reached in Wadbro and Berggren ${ }^{14}$ regarding the underdeterminedness of a problem with similar characteristics considered there. A number of comments and guidelines intended to help in using the proposed method are included in Appendix C.

\section{ACKNOWLEDGMENTS}

Professor Ole Sigmund at the Department of Mechanical Engineering at the Technical University of Denmark provided valuable aid and insight through several instructive and inspiring discussions. Assistant Engineer Jørgen Rasmussen at the Department of Electrical Engineering at the Technical University of Denmark helped to a large degree in preparing the experimental setup. The workshop at the Department of Mechanical Engineering at the Technical University of Denmark aided in the processing of select components for the experimental setup. The 3D-printing laboratory FabLab at the Technical University of Denmark produced the test specimens with Technician Jannick Schultz at the Department of Mechanical Engineering at the Technical University of Denmark in charge of the printing. The work was financially supported by Villum Fonden through the research project Topology Optimization - the Next Generation.

\section{APPENDIX A: DESIGN PROCEDURE DETAILS}

\section{Design problem}

The design problem is formulated as a min/max problem, where maximum value of $\Phi\left[p\left(f_{i}, \eta_{2, j}\right)\right]$, see Eq. (4), for $i \in\left\{1,2, \ldots, \mathcal{N}_{f}\right\}$ frequencies and $j \in\left\{1,2, \ldots, \mathcal{N}_{\eta_{2}}\right\}$ projection levels, is minimized over $\xi(\mathbf{x})$. That is, over the distribution of solid material in $\Omega_{\mathrm{d}, \mathrm{d}}$. Two constraints are considered. The first is that $p$ must be a solution to the model equations, Eqs. (1)-(3). The second is a volume constraint, imposed on the double filtered field, see Eq. (A2).

$$
\begin{aligned}
& \min _{\xi} \max _{f_{i}, \eta_{2, j}}\left(\Phi\left(p\left(\mathbf{x}, \hat{\tilde{\tilde{\tilde{\xi}}}}\left(\eta_{2, j}\right), f_{i}\right), p_{\text {target }}\left(\mathbf{x}, f_{i}\right)\right)\right), \\
& \text { s.t. } \left.\left.\frac{1}{\int \mathrm{d} \Omega_{\mathrm{d}}} \int \max _{\eta_{2, j}}\left(\hat{\tilde{\tilde{\xi}}}\left(\eta_{2, j}\right)\right) \mathrm{d} \Omega_{\mathrm{d}} \leq V, V \in\right] 0,1\right] .
\end{aligned}
$$

The min/max problem, Eqs. (A1) and (A2) is recast to the bound formulation proposed in Svanberg ${ }^{20}$ and solved using The Globally Convergent Method of Moving Asymptotes (GCMMA), ${ }^{20,24}$ with a maximum of three inner iterations per design iteration.

The following stopping criterion is used for the design procedure,

$$
\beta_{1}>80 \wedge \max _{m \in 1,2, \ldots, 5} \frac{\left|\Phi_{n}-\Phi_{n-m}\right|}{\left|\Phi_{n}\right|}<10^{-2},
$$

where $\Phi_{n}$ is the objective value at the $n$th iteration. To improve the convergence of the design procedure the problem is non-dimensionalized and the following rescaling and normalization is applied for $\rho, \kappa$, and $\omega$,

$$
(\hat{\rho}, \hat{\kappa})= \begin{cases}(1,1) & \text { for air } \\ \left(\frac{\rho_{\text {solid }}}{\rho_{\text {air }}}, \frac{\kappa_{\text {solid }}}{\kappa \text { air }}\right) & \text { for solid }, \hat{\omega}=\frac{\omega}{c} .\end{cases}
$$

\section{The design field}

During the design process, the design itself is modeled as a distribution of solid material in an air background within $\Omega_{\mathrm{d}}$. The auxiliary design field, $\xi$, controls the distribution by interpolating the inverse material parameters, $\rho^{-1}$ and $\kappa^{-1}$ between solid material $(\xi=1)$ and air $(\xi=0)$. The introduction of $\xi$ allows for a mix of solid material and air to exist at each point in space during the intermediate stages of the design process. This mix is gradually removed by applying a projection scheme with a continuation approach on the projection strength as part of the design process. ${ }^{25,26}$ The bounds on $\xi$ are defined as: $\xi(\mathbf{x}) \in[0,1] \forall \mathbf{x} \in \Omega_{\mathrm{d}, \mathrm{d}}, \xi(\mathbf{x})$ $=0 \forall \mathbf{x} \in \Omega \backslash\left(\Omega_{\mathrm{d}} \backslash \Omega_{\mathrm{d}, \mathrm{e}}\right), \xi(\mathbf{x})=1 \forall \mathbf{x} \in \Omega_{\mathrm{d}, \mathrm{f}}$.

The proposed design method allows for designing devices which perform robustly under geometric perturbations, like what might occur during production, installation or use. For the considered cases only near-uniform geometric perturbations are included, ${ }^{27}$ where the size of all features in the 
design device is varied in a near-identical manner. However non-uniform spatial perturbations of the designs can also be considered. ${ }^{28}$ The robustness is assured by using a double filtering technique, ${ }^{18}$ consists of applying a smoothing operator, ${ }^{29,30}$

$$
\begin{aligned}
& \tilde{\xi}\left(\mathbf{x}_{i}\right)=\frac{\int_{\Omega_{\mathrm{d}}} w\left(\mathbf{x}_{i}-\mathbf{x}_{j}\right) \xi\left(\mathbf{x}_{j}\right) \mathrm{d} \mathbf{x}_{j}}{\int_{\Omega_{\mathrm{d}}} w\left(\mathbf{x}_{i}-\mathbf{x}_{j}\right) \mathrm{d} \mathbf{x}_{j}}, \\
& w(\mathbf{x})= \begin{cases}R-|\mathbf{x}| & \forall|\mathbf{x}| \leq R \wedge \mathbf{x} \in \Omega_{\mathrm{d}} \\
0 & \text { otherwise },\end{cases}
\end{aligned}
$$

where $R$ is a filter radius, followed by a projection operator, $^{27}$

$$
\hat{\xi}(\xi)=\frac{\tanh (\beta \eta)+\tanh (\beta(\xi-\eta))}{\tanh (\beta \eta)+\tanh (\beta(1-\eta))},
$$

where $\eta$ is the projection level and $\beta$ is the projection strength, to $\xi$ twice.

The double filter requires two filter radii, $R_{1}=2 R_{2}$ as well as two projection strengths $\beta_{1}=2 \beta_{2}$ and two projection levels $\eta_{1}, \eta_{2}$. A continuation scheme on the projection strength is applied on $\beta_{1}$ with $\beta_{1}=\beta_{\text {ini }}$ as the initial value and this value is then increased by a factor of 1.2 every 50 design iterations or when the objective function value changes by less than $1 \%$ over 5 consecutive iterations. This procedure is continued until $\beta_{1} \geq 80$ is reached. At this value for $\beta_{1}$ the distribution of solid material and air resulting from the design process is close to being discrete throughout $\Omega_{\mathrm{d}}$, limiting the need for a postprocessing step. The application of the double filter on $\xi$ means that the interpolation of $\rho^{-1}$ and $\kappa^{-1}$ in Eq. (A8) is performed using the doubly filtered field as,

$$
\begin{gathered}
\rho^{-1}=\rho_{\text {air }}^{-1}+\hat{\tilde{\tilde{\tilde{\xi}}}}\left(\rho_{\text {solid }}^{-1}-\rho_{\text {air }}^{-1}\right), \\
\kappa^{-1}=\kappa_{\text {air }}^{-1}+\hat{\tilde{\tilde{\tilde{\xi}}}}\left(\kappa_{\text {solid }}^{-1}-\kappa_{\text {air }}^{-1}\right) .
\end{gathered}
$$

For the software implementation, the doubly filtered auxiliary field is discretized into a piecewise constant field consisting of $\mathcal{N}_{\mathrm{e}}$ discrete design variables coinciding with the finite element discretization of $\Omega_{\mathrm{d}}$. The sensitivities of the objective function with respect to the design variables, $\mathrm{d} \Phi / \mathrm{d} \xi_{j}, j \in\left\{1,2, \ldots, \mathcal{N}_{\mathrm{e}}\right\}$, needed for solving the optimization problem using GCMMA, are calculated using adjoint sensitivity analysis. ${ }^{31}$

\section{APPENDIX B: EXPERIMENTAL SETUP DATA}

See Table II.
TABLE II. Dimensions of components used in the experimental setup along with material types and parameter values. An upper bound on the uncertainty is included where deemed relevant.

\begin{tabular}{lc}
\hline \hline Component & Width $\times$ Breadth $\times$ Height \\
\hline Chamber: & \\
Chamber, [m] & $0.785 \times 0.785 \times 0.006 \pm 5 \times 10^{-4}$, \\
PVC (base) plate [m] & $1.1 \times 1.1 \times 0.01$ \\
Acrylic (top) plate [m] & $1.5 \times 1.5 \times 0.01$ \\
Wooden strips [m] & $1.1 \times 0.026 \times 0.006 \pm 5 \times 10^{-4}$ \\
Absorbing foam strips [m] & $1.05 \times 0.130 \times 0.006 \pm 5 \times 10^{-4}$ \\
Test specimen: & $(0.2 \times 0.1 \times 0.01) \pm 2 \times 10^{-4}$ \\
Base [m] & $(0.1 \times 0.05 \times 0.006) \pm 2 \times 10^{-4}$ \\
Device [m] & value or type \\
Material: & Basotec from BASF \\
Absorbing foam: & Trovidur EC \\
PVC Plate: & Plexiglas XT \\
Acrylic Plate: & 954 \\
ABS plastic: & 1.9 \\
- Density, $\rho\left[\mathrm{kg} / \mathrm{m}^{3}\right]$ & \\
- Bulk Modulus, $\kappa[\mathrm{GPa}]$ & \\
\hline \hline
\end{tabular}

\section{APPENDIX C: COMMENTS AND GUIDELINES}

During the development and testing of the proposed method it was found that special care should be exercised regarding the points listed below.

(1) The energy contained in $p_{\text {target }}$ must be equal to the energy emitted by the sources to obtain a high transmission of energy from the device. For example, if more energy is emitted from the sources than are present in $p_{\text {target }}$ it is impossible to achieve full transmission of the energy from the sources into $p_{\text {target }}$.

(2) Select a correct scaling of optimization parameters. Tuning may be needed to assure smooth convergence for the problem.

(3) The frequency bandwidth for which the device should operate and the size of the geometric perturbations are important to the convergence behavior of the design procedure, as well as for the performance of the final designs. For example, if an overly wide frequency band is considered the resulting design may perform poorly and/or the optimization algorithm may have problems converging.

${ }^{1}$ M. Bendsøe and O. Sigmund, Topology Optimization, 2nd ed. (SpringerVerlag, Berlin, Germany, 2004), 370 pp.

${ }^{2}$ J. Chang and F. Jacobsen, "Sound field control with a circular doublelayer array of loudspeakers," J. Acoust. Soc. Am. 131(6), 4518-4525 (2012).

${ }^{3}$ J. Chang and F. Jacobsen, "Experimental validation of sound field control with a circular double-layer array of loudspeakers," J. Acoust. Soc. Am. 133(4), 2046-2054 (2013).

${ }^{4}$ M. Ureda, "Analysis of loudspeaker line arrays," J. Audio Eng. Soc. 52(5), 467-495 (2004).

${ }^{5} \mathrm{M}$. McKemie and C. McKinney, "An experimental investigation of the parabolic reflector as a nearfield calibration device for underwater sound transducers," J. Acoust. Soc. Am. 67, 523-529 (1980).

${ }^{6} \mathrm{~T}$. Hueter, "Twenty years in underwater acoustics: Generation and reception," J. Acoust. Soc. Am. 51(3), 1025-1040 (1972).

${ }^{7}$ S. Wahlström, "The parabolic reflector as an acoustical amplifier," J. Audio Eng. Soc. 33(6), 418-429 (1985). 
${ }^{8}$ R. van der Rots and A. Berkhoff, "Directional loudspeaker arrays for acoustic warning systems with minimised noise pollution," Appl. Acoust. 89, 345-354 (2015).

${ }^{9}$ M. Bai, J. Ih, and J. Benestry, Acoustic Array Systems: Theory, Implementation, and Application (John Wiley and Sons, Singapore, 2013), $510 \mathrm{pp}$.

${ }^{10}$ M. Clark, "An acoustic lens as a directional microphone," J. Acoust. Soc. Am. 25, 1152-1153 (1953).

${ }^{11}$ R. Little, "Acoustic properties of parabolic reflectors," J. Acoust. Soc. Am. 40, 919-920 (1966).

${ }^{12}$ F. Jacobsen and P. Juhl, Fundamentals of General Linear Acoustics (John Wiley and Sons, Weinheim, Germany, 2013), Chaps. 6 and 9.

${ }^{13}$ E. Bängtsson, D. Noreland, and M. Berggren, "Shape optimization of an acoustic horn," Comput. Methods Appl. Mech. Eng. 192, 1533-1571 (2003).

${ }^{14} \mathrm{E}$. Wadbro and M. Berggren, "Topology optimization of an acoustic horn," Comput. Methods Appl. Mech. Eng. 196, 420-436 (2006).

${ }^{15}$ M. Dühring, J. Jensen, and O. Sigmund, "Acoustic design by topology optimization,” J. Sound Vib. 317, 557-575 (2008).

${ }^{16} \mathrm{~J}$. Du and N. Olhoff, "Minimization of sound radiation from vibrating bimaterial structures using topology optimization," Struct. Multidisciplinary Optim. 33, 305-321 (2007).

${ }^{17}$ J. Kook, K. Koo, J. Hyun, J. Jensen, and S. Wang, "Acoustical topology optimization for zwicker's loudness model - application to noise barriers," Comput. Methods Appl. Mech. Eng. 237, 130-151 (2012).

${ }^{18}$ R. Christiansen, B. Lazarov, J. Jensen, and O. Sigmund, "Creating geometrically robust designs for highly sensitive problems using topology optimization - acoustic cavity design," Struct. Multidisciplinary Optim. 52, 737-754 (2015).

${ }^{19}$ R. Christiansen, E. Fernandez-Grande, and O. Sigmund, "Experimental validation of a topology optimized acoustic cavity," J. Acoust. Soc. Am. 138(6), 3470-3474 (2015).
${ }^{20} \mathrm{~K}$. Svanberg, "A class of globally convergent optimization methods based on conservative convex seperable approximations," SIAM J. Optim. 12, 555-573 (2002)

${ }^{21}$ R. Udawalpola and M. Berggren, "Optimization of an acoustic horn with respect to efficiency and directivity," Int. J. Numer. Methods Eng. 73, 1571-1606 (2007).

${ }^{22}$ B. Hal, W. Desmet, B. Pluymers, P. Sas, and D. Vandepitte, "A coupled finite element - wave based approach for the steady-state dynamics analysis of acoustic systems," J. Comput. Acoust. 11, 285-303 (2003).

${ }^{23} \mathrm{~B}$. Pluymers, "Wave based modelling methods for steady-state vibroacoustics," Ph.D. thesis, Katholieke Universiteit Leuven, Leuven, Belgium, 2006, Chaps. 6 and 7, 342 pp.

${ }^{24} \mathrm{~K}$. Svanberg, "The method of moving asymptotes - a new method for structural optimization," Int. J. Numer. Methods Eng. 24, 359-373 (1987).

${ }^{25}$ J. Guest, J. Prévost, and T. Belytschko, "Achieving minimum length scale in topology optimization using nodal design variables and projection functions,” Int. J. Numer. Methods Eng. 61, 238-254 (2004).

${ }^{26} \mathrm{~S}$. Xu, Y. Cai, and G. Cheng, "Volume preserving nonlinear density filter based on heaviside projections," Struct. Multidisciplinary Optim. 41, 495-505 (2010).

${ }^{27}$ F. Wang, B. Lazarov, and O. Sigmund, "On projection methods, convergence and robust formulations in topology optimization," Struct. Multidisciplinary Optim. 43, 767-784 (2011).

${ }^{28}$ M. Schevenels, B. Lazarov, and O. Sigmund, "Robust topology optimization accounting for spatially varying manufacturing errors," Comput. Methods Appl. Mech. Eng. 200, 3613-3627 (2011).

${ }^{29}$ B. Bourdin, "Filters in topology optimization," Int. J. Numer. Methods Eng. 50, 2143-2158 (2001).

${ }^{30} \mathrm{~T}$. Bruns and D. Tortorelli, "Topology, optimization of non-linear elastic structures and compliant mechanisms," Comput. Methods Appl. Mech. Eng. 190, 3443-3459 (2001).

${ }^{31}$ D. Tortorelli and P. Michaleris, "Design sensitivity analysis: Overview and review," Inverse Problems Eng. 1, 71-103 (1994). 